\title{
Association of Physical Activity with Psychological and Biological Markers of Mental Health in an Internet-based Health Promotion Program: A Pilot Study
}

\author{
Hideki Kamikawa ${ }^{1)}$, Yasuki Higaki ${ }^{2)}$, Miwa Tayama ${ }^{3)}$, Guangying $\mathrm{Li}^{4)}$, \\ Naoto Taguchi ${ }^{5)}$, Keitaro Tanaka ${ }^{5)}$, Shigeto Yamada ${ }^{4)}$ \\ ${ }^{1)}$ Department of Psychiatry, Keiaikai Nakagami Hospital \\ ${ }^{2}$ Department of Health and Exercise Science, Faculty of Sports and Health Science, Fukuoka University \\ ${ }^{3)}$ Mental Health Care Section, Keiaikai Nakagami Hospital \\ 4) Department of Psychiatry, Faculty of Medicine, Saga University \\ ${ }^{5)}$ Department of Preventive Medicine, Faculty of Medicine, Saga University
}

\begin{abstract}
The objective was to assess the mental health of the participants using psychological and biological indicators in a health promotion program. We conducted the individual program without any face-to-face communications among instructors and participants. The program offered Physical Activity (PA) data and exercise guidance to subjects, using digital devices. We quantified the amount and intensity of daily PA with a uniaxial accelerometer. Activities are categorized into eleven activity levels $(0,0.5,1.0-9.0)$. Two batteries of psychosocial questionnaires and measurements of free-3-methoxy-4-hydroxyphenylglycol (MHPG) in saliva were completed before and after the program. The program showed no significant changes of mean values of all subjects in the Beck Depression Inventory (BDI) and General Health Questionnaire (GHQ) scores, and the amounts of MHPG, either. A positive correlation was observed between the increases in the PA and the reductions in the BDI score. Furthermore, the BDI reductions correlated positively with the increased time spent in the PA at light and moderate activity levels. The MHPG increases demonstrated a significant relationship with the decreases of time spent in the PA at the activity level of sleep or non-activity. The different properties of MHPG as an index of mental health were compared with psychological questionnaires. This pilot study suggested that monitoring not only the total amounts of PA but also the intensity and duration of activities might be important to offer adequate guidance of mental health to individuals. Further research is needed to evaluate the impact of PA on mental health.
\end{abstract}

Key words: free-3-methoxy-4-hydroxyphenylglycol, physical activity, internet, depression, accelerometry sensor, health promotion, information technology

\section{Introduction}

The merits of physical activity (PA) as a standard therapy for life-style-associated diseases, such as obe-

Received: July 28, 2010

Accepted: December 15, 2010

Correspondence: H. Kamikawa, Department of Psychiatry, Keiaikai Nakagami Hospital, 6-25-5 Chibana, Okinawa 9042195, Japan

e-mail:kamikawa@nakagami.or.jp sity, diabetes, and cardiovascular diseases, have been well demonstrated. Recently, the focus of PA has shifted to its positive effect on mental health. One study reported that aerobic exercise at a dose consistent with public health recommendations is an effective treatment for depression ${ }^{1}$. In contrast, exercise over the lactate threshold (LT) has been shown to activate the hypothalamic-pituitary-adrenal (HPA) axis while increasing the plasma corticotropin (ACTH) and cortisol levels in the blood ${ }^{2}$. This response leads to adverse 
events, including the "over-training syndrome", insomnia, loss of appetite, and depression ${ }^{3)}$. It is important to monitor the amount of PA and the mental states of participants during a health promotion program.

Traditionally, PA has been assessed using questionnaires, but there are limitations in subjects' recall ability, especially for ubiquitous, light, or moderateintensity activities ${ }^{4}$. The activity monitor under freeliving conditions based on a uniaxial accelerometry sensor is capable of obtaining objective information on PA patterns, since it can continuously measure steps, and the intensity, duration and frequency of activities. Based on the results from the comparison of 13 models of pedometers, the electronic pedometer Kenz Lifecorder (Suzuken Co. Ltd, Nagoya, Japan) is available for applied physical activity research ${ }^{4}$.

The Beck Depression Inventory (BDI) and the General Health Questionnaire (GHQ) have been widely used for assessing psychiatric symptoms of subjects $^{5,6)}$. Like other questionnaires, the BDI and GHQ can be exaggerated or minimized by specific situations, such as the way they are administered or the complications of concomitant physical illnesses ${ }^{7,8)}$. Therefore, biological indicators are needed to assess mental health.

Free-3-methoxy-4-hydroxyphenylglycol (MHPG) is a major metabolite of noradrenaline in the human brain. Several reports indicated that healthy volunteers with job stress show a high plasma level of $\mathrm{MHPG}^{9,10)}$. It has been reported that the saliva level of MHPG correlates significantly with plasma and cerebrospinal fluid levels of the metabolite ${ }^{11,12}$. We previously reported that the saliva level of MHPG was strongly correlated with the plasma MHPG level in normal individuals ${ }^{13)}$. Because the sampling procedure for saliva is much less invasive than that for blood drawing, the free saliva MHPG level can be a useful indicator for assessing not only the level of psychiatric symptoms, but also the response to treatment in clinical practice.

To evaluate the mental health of all participants in the comprehensive health promotion program, two questionnaires were administered before and after the program. At the same time, the MHPG concentrations in the saliva were also measured as a biological index. The daily amount and intensity of PA in an individual setting was quantified using a uniaxial accelerometer. The hypothesis of this pilot study was that PA would reduce both depressive symptoms and the MHPG levels as a biological stress marker.

\section{* Methods}

\section{Procedure}

The program was conducted for 4 months from October 2004 to February in 2005. This study used a pre/post measure design, which used subjects as their own controls. All subjects were mentally and physically assessed on two occasions: before and after the program. We estimated their mental health levels using two batteries of psychosocial questionnaires and MHPG measurement. Depressive symptoms were assessed by Beck Depression Inventory II (BDI). Psychiatric symptoms and general distress symptoms were assessed by the 28-item General Health Questionnaire (GHQ). We continuously monitored the PA between the former week before the start of the program and the final week of the program. The daily average value of PA during a week was calculated as the daily PA.

\section{Subjects}

Twenty-six Healthy Japanese subjects (41-59 yr old) recruited by the Saga City Office participated in this study. After this study was carefully explained, each subject signed an informed consent statement. People who were unable to use IT devices were excluded before registrations. The study protocol was approved by the ethical guidelines of Saga City in accordance with the Helsinki Declaration.

\section{Instrument}

We measured the PA using a uniaxial accelerometer (Kenz Lifecorder ${ }^{\mathbb{R}}$ EX; Suzuken Co. Ltd, Nagoya, Japan). It measured $7 \times 4 \times 1.5 \mathrm{~cm}$ in size and was rigidly fixed on the belt during the daytime. The activity monitor measures the acceleration in the vertical direction. According to the technical details provided by the manufacturer (Suzuken Co. Ltd, Nagoya, Japan), the activities are categorized into eleven activity levels $(0,0.5$ and $1.0-9.0$; level) based on the pattern of the accelerometric signal. The activity levels are subsequently converted by an algorithm to calculate the energy expenditure (EE). This accelerometer accurately assessed EE (kcal) during both the exercise period and non-structured activities ${ }^{14}$. Activity level 1.0, 4.0, 7.0 are equal to 1.8, 3.6, 6.1 MET (metabolic equivalents), respectively. 


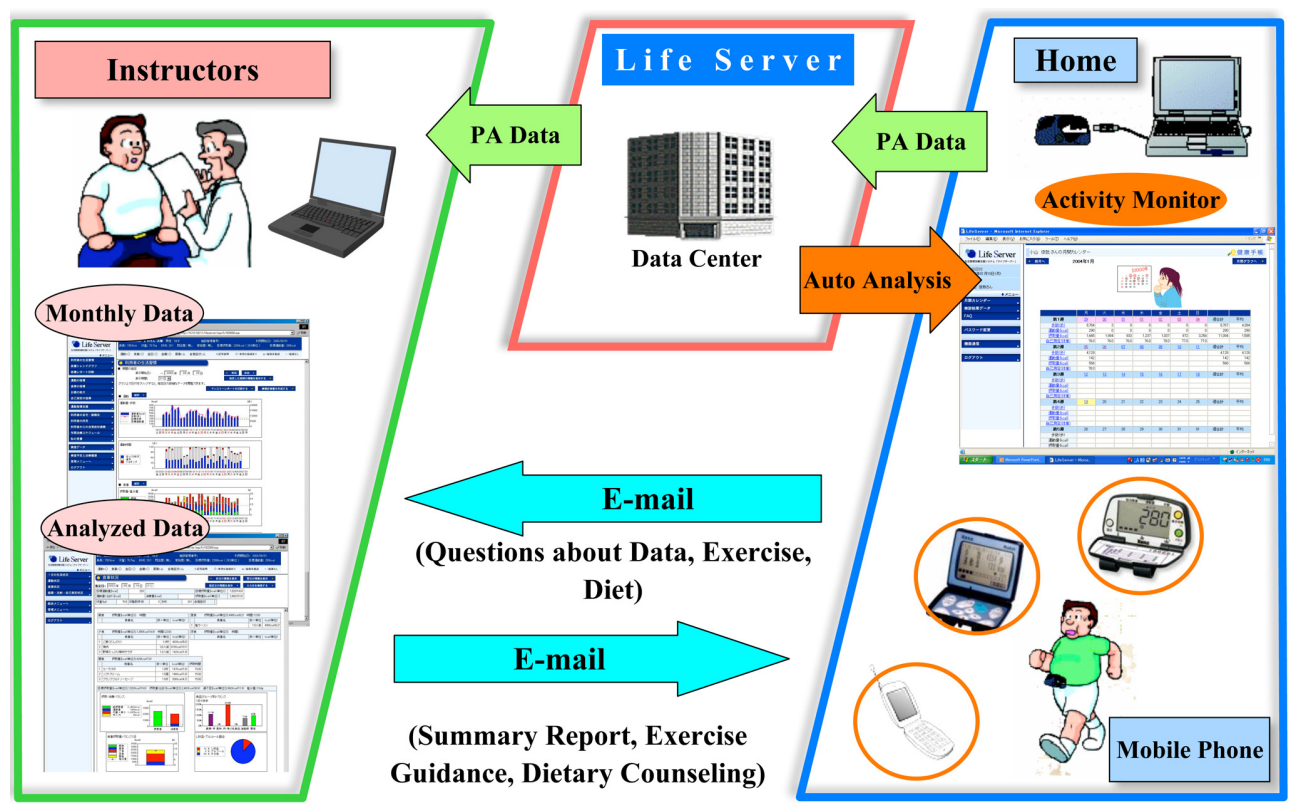

Figure 1 The illustration of the internet-based health promotion program.

CCopyright 2006-2007 Suzuken Company Limited. All Rights Reserved.

\section{Health promotion welb page}

The subjects are remotely supported by instructors using information technology (IT) devices: i.e., internet, e-mail. The data, including the total EE, total step frequencies and raw data based on an accelerometer, can be stored for 200 days. The data are then transmitted to the Suzuken's web page (Health Diary). Suzuken then supplies advice directly to individuals concerning their progress regarding specific targets and methods to lead healthier lifestyles. The subjects can download their summary via their personal computer and then communicate with instructors by email if they so desire (Figures 1 and 2).

MHPG measurement. We measured the MHPG concentrations in the saliva before and after the program. For the collection of saliva, a sterile cotton swab was chewed by a participant for $2 \mathrm{~min}$. The recovery of saliva from the cotton swab was carried out by centrifugation at $3,500 \mathrm{rpm}$ for $20 \mathrm{~min}$ and 0.5 $\mathrm{ml}$ of saliva from each individual was then placed in a tube and stored at $-80^{\circ} \mathrm{C}$. MHPG in the saliva was determined by high performance liquid chromatography with electrochemical detection according to the method of Minegishi and Ishizaki ${ }^{15)}$. MHPG in saliva was extracted employing a small $\mathrm{C} 18$ column. Nanillyl alcohol was used as an internal standard. The data were expressed $\mathrm{ng} / \mathrm{ml}$.

Expression of the data and statistical analyses.
The changes in BDI and GHQ in the program were evaluated by the Wilcoxon signed-ranks test. The changes in PA, EE and MHPG were examined by the paired Student's $t$ test. The Spearman's correlation coefficient by rank was calculated to examine the correlation of changes in the PA with changes in the BDI and GHQ score, and MHPG, and changes in the time spent in PA at each intensity level with changes in the BDI and GHQ, and MHPG. A $p$ value of $<0.05$ was thus considered to be statistically significant. All data are presented as the means $\pm \mathrm{SD}$.

\section{Results}

A total of 24 male and 2 female subjects were eligible for the study. Twenty-two male and 1 female subjects completed the study. The demographic characteristics, including age, baseline PA, EE, BDI, GHQ and MHPG are shown in Table 1.

The program demonstrated no significant changes in the PA $(\mathrm{t}(22)=-0.25, \mathrm{p}=0.81)$. The changes in the daily PA of each subject varied from $-5,667$ steps $(-128.9 \mathrm{kcal})$ to $+6,350$ steps $(+219.3$ kcal).

This program tended to reduce the BDI scores from 7.1 (ranges: 0 to $15 ; \mathrm{SD}=5.2$ ) to 5.4 (ranges: 0 to 18; $\mathrm{SD}=5.0$ ), while increasing the GHQ scores from 3.7 (ranges: 0 to $11 ; \mathrm{SD}=3.4$ ) to 4.4 (ranges: 0 to 17 ; 


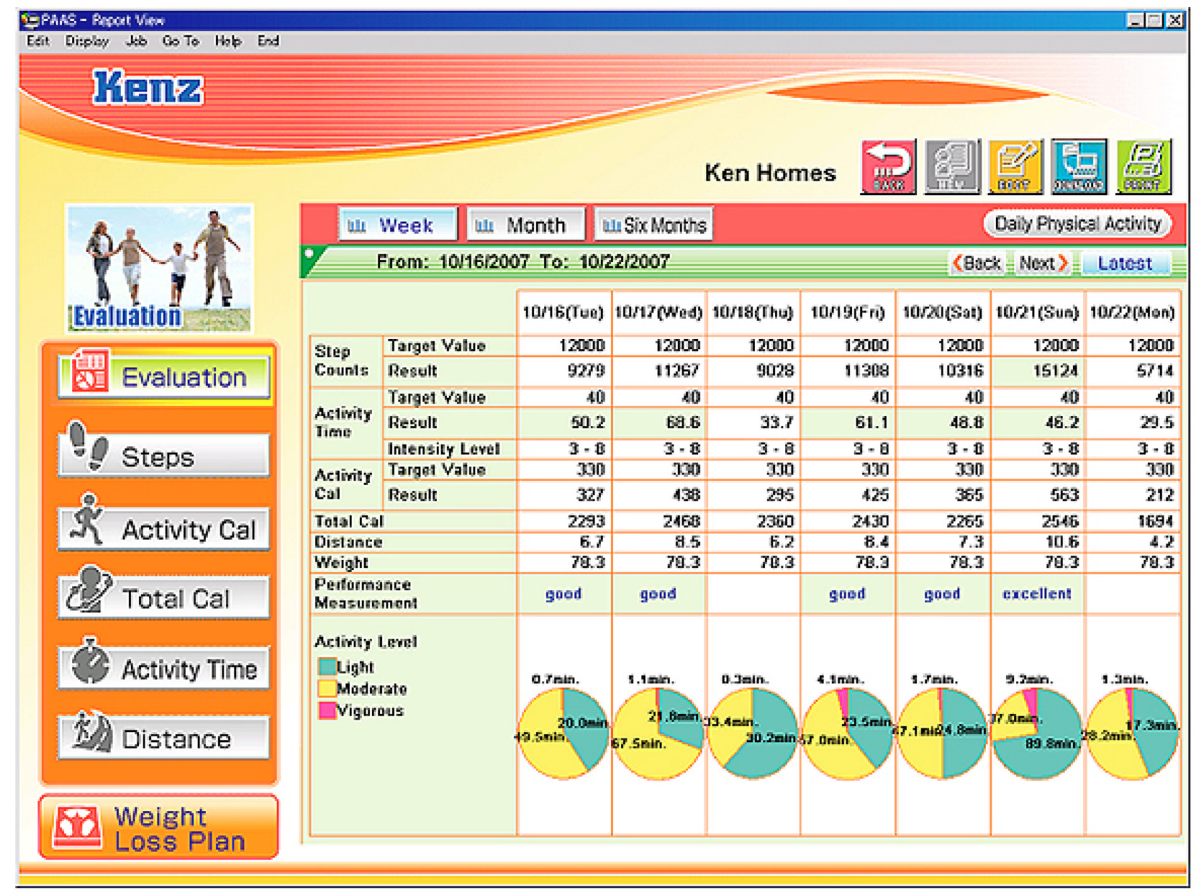

Figure 2 The program offered PA data and exercise guidance as a Log Book to subjects. CCopyright 2006-2007 Suzuken Company Limited. All Rights Reserved.

$\mathrm{SD}=4.5)$, however, no significant difference was found $(\mathrm{z}=-1.81, \mathrm{p}=0.07)(\mathrm{z}=-0.40, \mathrm{p}=0.69)$. There was a positive relationship between the increases in the $\mathrm{PA}$ and the reductions in the BDI ( $\mathrm{rho}=-0.449$, $\mathrm{p}=0.035$ ) (Figure 3, Table 2). Furthermore, the BDI reductions correlated positively with the increases of duration in the PA at the activity levels of 2,3 , and 4 indicating the light to moderate activity (corresponding to walking or running slowly) (rho $=-0.454$, $\mathrm{p}=0.033)(\mathrm{rho}=-0.494, \mathrm{p}=0.021) \quad(\mathrm{rho}=-0.429$, $\mathrm{p}=0.044)$. On the other hand, the MHPG changes had no correlation with the changes of time spent in the PA at the consistent intensity levels (Table 3$)$ (rho $=0.378$, $\mathrm{p}=0.076)(\mathrm{rho}=0.126, \mathrm{p}=0.554)(\mathrm{rho}=0.182, \mathrm{p}=0.392)$.

The MHPG increases in the saliva demonstrated a significant relationship with the increases of duration in the PA at the activity levels of 0.5 and 1 (correspond to 1 to 1.8 Mets, studying or driving) ${ }^{14)}$ (rho $=0.673$, $\mathrm{p}=0.002)(\mathrm{rho}=0.431, \mathrm{p}=0.043)$ and with the decrease of duration in the PA at the 0-intensity level (sleep or non-activity) (Table 3) (rho $=0.591, \mathrm{p}=0.006)$.

\section{Discussion}

This study revealed a positive correlation between the decreased BDI and PA increases in an
Table 1 Characteristics of subjects.

\begin{tabular}{lcc}
\hline & Baseline & After Program \\
\hline Age (yr) & $50.5(6.8)$ & \\
BDI score & $7.1(5.2)$ & $5.4(5.0)$ \\
GHQ score & $3.7(3.4)$ & $4.4(4.5)$ \\
MHPG (ng/ml) & $15.4(17.9)$ & $10.8(12.4)$ \\
PA (steps/day) & $8,243(3,229)$ & $8,102(3,161)$ \\
EE (kcal/day) & $253(114)$ & $261(112)$ \\
\hline
\end{tabular}

$\mathrm{n}=23$, Data are mean (SD). SD: Standard Deviation.

No signicant differences were observed in all indices between baseline and after program (the Wilcoxon signed-ranks test or the paired Student's $t$ test).

internet-based health promotion program. Reductions in the BDI and/or GHQ scores showed a significant relationship to the PA at the light and moderate intensity levels (equivalent to walking or running slowly). Contrary to the previous hypothesis, the increase in MHPG in the saliva demonstrated a significant relationship with a decrease in the duration of PA at the 0 -intensity level (sleep or non-activity). These results suggested the different properties of MHPG as an index of mental health in comparison to psychological questionnaires.

We previously reported that the saliva levels of 


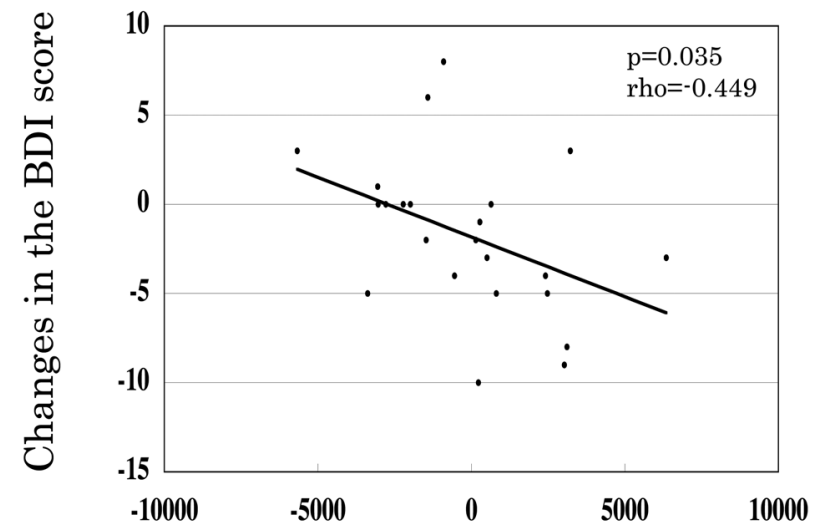

Changes in the Physical Activity (steps)

Figure 3 The relationship between changes in the PA and the BDI scores in the health promotion program.

MHPG in patients with anxiety disorders were significantly higher than those of control subjects, and that the levels were reduced by $1 \mathrm{wk}$ of alprazolam treatment ${ }^{16)}$. Furthermore, several reports have indicated that healthy volunteers with job stress show a high plasma level of $\mathrm{MHPG}^{9,10)}$. These studies suggested that increased MHPG could indicate deleterious states of mental health. The hypothesis of our pilot study was that participants who increased PA would reduce both their depressive symptoms and MHPG levels. In contrast with the depressive symptoms, our present investigation demonstrated that the MHPG changes had no correlation with the changes of time spent in the PA at the light and moderate intensity. These inconsistent results between the BDI and MHPG are thus considered to weaken support for the above hypothesis on the effects of our program.

McEwen's theory on the stress response is useful in explaining the conflicting results of the current study ${ }^{17,18)}$. A process in which the internal milieu varies to meet perceived and anticipated demand, which has been referred to as allostasis by McEwen. The NA
Table 2 Correlations between changes in the PA and changes in the BDI, GHQ and MHPG after the completion of health promotion program

\begin{tabular}{lccc}
\hline & BDI score & GHQ score & MHPG \\
\hline PA (steps) & $-0.449^{*}$ & -0.263 & 0.129 \\
\hline
\end{tabular}

Data are rho value. *: $\mathrm{p}<0.05$ (the Spearman's correlation coefficient by rank).

responses to an event initiating a novel life-style may be adaptive in the short run. However, if recovery from the acute event is not accompanied by an adequate homeostatic response to terminate the acute adaptive response of the NA system, then deleterious effects on the psychological and physiological function, termed the allostatic load, might occur. Based on our present results, we speculated that the increases of PA at light and moderate intensity levels might play a role in improving depressive symptoms, and that the increased state of arousal might facilitate the NA activation, potentially leading to a deleterious outcome to the participants in the long run. McEwen's concept of stress responses shows a possible link between the protective value of BDI reduction during the shortterm, and the adverse consequences of NA activation for subjects in the long-term.

Exercise over the LT has been shown to activate the HPA axis while increasing the ACTH and cortisol levels in the blood ${ }^{2}$. This response might lead to allostatic states, or an "over-training syndrome", insomnia, loss of appetite loss, and depression ${ }^{3)}$. We previously reported that mild exercise at the LT for 6 wk had a preventative effect on glucose intolerance ${ }^{19}$. As our exercise guidance in this program, we recommended a standard heart rate equivalent to less than the estimated LT as a safe and effective exercise load to prevent life-style-associated diseases. This exercise load might also be effective for preventing partic-

Table 3 Correlations between changes in the time spent in each intensity level activity and changes in the BDI, GHQ and MHPG after the completion of health promotion program

\begin{tabular}{lccccccccccc}
\hline & \multicolumn{10}{c}{ Activity levels } \\
\cline { 2 - 14 } & 0 & 0.5 & 1 & 2 & 3 & 4 & 5 & 6 & 7 & 8 & 9 \\
\hline BDI score & 0.398 & -0.306 & -0.343 & $-0.454^{*}$ & $-0.494^{*}$ & $-0.429^{*}$ & -0.289 & -0.272 & -0.218 & -0.156 & -0.190 \\
GHQ score & 0.046 & 0.110 & -0.107 & -0.036 & -0.097 & $-0.421^{*}$ & -0.118 & -0.272 & -0.117 & 0.000 & 0.223 \\
MHPG (ng/ml) & $-0.591^{* *}$ & $0.673^{* *}$ & $0.431^{*}$ & 0.378 & 0.126 & 0.182 & 0.065 & 0.219 & -0.031 & 0.024 & 0.121 \\
\hline
\end{tabular}

Data are rho value. *: $\mathrm{p}<0.05{ }^{* *}: \mathrm{p}<0.01$ (the Spearman's correlation coefficient by rank). 
ipants from demonstrating the symptoms of allostatic load and thereby establishing an allostatic state. Our pilot study suggested that quantifying the intensity and duration of activities might be important to offer adequate guidance to improve mental health of individuals.

There were several limitations in our study that should be noted. First, as the present investigation did not include a control group, it is difficult to evaluate the impact of PA on mental health in the observed outcomes. Second, our intervention did not significantly increase the mean values of PA of all subjects. This result indicated that social support in a health promotion program might be a critical factor to promote exercise and physical activities. Another possible reason for the intervention inefficiency was that the present results might have been influenced by seasonal variations, including the climate and social factors. We quantified PA as a pre-control in October. In this month, many athletic festivals and sports events were held all over the country because Health and Sports Day (a national holiday) is held on the second Monday in October. According to the Japan Meteorological Agency, the average temperature in this location was 23.6 degrees Celsius (74.5 degrees Fahrenheit) in October and 6.3 degrees Celsius (43.3 degrees Fahrenheit) in February, at the end of the program. Third, IT literacy might have influenced the results of our study because participants were required to use digital devices to enroll in the program. Applicants who were unable to use IT devices were excluded before registration. Therefore, the number of female participants was lower than that of male participants.

\section{* Conclusions}

The mental health of subjects in an internet-based individual health promotion program was assessed. The different properties of MHPG as an index of mental health were compared with psychological questionnaires. This pilot study suggested that monitoring not only the total amounts of PA, but also the intensity and duration of activities, might be important to offer the adequate guidance of mental health to individuals. Further research and randomized controlled trials are needed to evaluate the impact of PA on mental health.

\section{Acknowledgements}

This study was conducted by the Saga City Office as part of the health promotion campaign (Healthy Saga 21) which was financially supported by Suzuken Co. Ltd.

\section{References}

1) Dunn AL, Trivedi MH, Kampert JB, Clark CG, Chambliss HO: Exercise treatment for depression efficacy and dose response. Am J Prev Med 28, 1-8 (2005).

2) Saito T, Soya H: Delineation of responsive AVPcontaining neurons to running stress in the hypothalamus. Am J Physiol Regul Integr Comp Physiol 286, R484-490 (2004).

3) Kuiper H, Keizer HA: Overtraining in elite athletes. Sports Med 6, 79-92 (1988).

4) Beck AT, Steer RA, Ball R, et al.: Comparison of beck depression inventories -IA and -II in psychiatric outpatients. J Pers Assess 67, 588-597 (1996).

5) Goldberg DP, Hillier VF: A scaled version of the General Health Questionnaire. Psychol Med 9, 139145 (1979).

6) Bowling A: Mode of questionnaire administration can have serious effects on data quality. J Public Health (Oxf) 27, 281-291 (2005).

7) Moore MJ, Moore PB, Shaw PJ: Mood disturbances in motor neuron disease. J Neurol Sci 160 (Suppl 1), S53-56 (1998).

8) Maas JW, Hattox SE, Landis DH, Roth RH: The determination of a brain arteriovenous difference for 3-methoxy-4-hydroxyphenethyleneglycol (MHPG). Brain Res 118, 167-173 (1976).

9) Drebing CJ, Freedman R, Waldo M, Gerhardt GA: Unconjugated methoxylated catecholamine metabolites in human saliva. Quantitation methodology and comparison with plasma levels. Biomed Chromatogr 3, 217-220 (1989).

10) Reuster $T$, Rilke $O$, Ochler J: High correlation between salivary MHPG and CSF MHPG. Psychopharmacology (Berl) 162, 415-418 (2002).

11) Yajima J, Tsuda A, Katayanagi $K$, et al.: Measurement of saliva free-3methoxy-4-hydroxyphenyulethyleneglcol and effects of stress. J Brain Sci 23, 321-322 (1998).

12) Yamada S, Yajima J, Harano M, et al.: Saliva level of free-3methoxy-4-hydroxyphenylglycol in psy- 
chiatric outpatients with anxiety. Int Clin Psychopharmacol 13, 213-217 (1998).

13) Lundberg U, Frankenhaeuser M: Stress and workload of men and women in high-ranking positions. J Occup Health Psychol 4, 142-151 (1999).

14) Mitoma M, Yoshimura R, Sugita A, Umene $W$, Hori H, Nakano H, et al.: Stress at work alters serum brain-derived neurotrophic factor (BDNF) levels and plasma 3-methoxy-4-hydroxyphenylglycol (MHPG) levels in healthy volunteers: BDNF and MHPG as possible biological markers of mental stress? Prog Neuropsychopharmacol Biol Psychiatry 32, 679-685 (2008).

15) Kumahara H, Schutz Y, Ayabe M, Yoshioka M, Yoshitake Y, Shindo M, et al.: The use of uniaxial accelerometry for the assessment of physical-activity-related energy expenditure: a validation study against whole-body indirect calorimetry. Br J Nutri- tion 91, 235-243 (2004).

16) Minegishi A, Ishizaki T: Determination of free 3methoxy-4-hydroxyphenylglycol with several other monoamine metabolites in plasma by high-performance liquid chromatography with amperometric detection. J Chromatogr 311, 51-57 (1984).

17) McEwen BS: Allostasis and allostatic load: implications for neuropsychopharmacology. Neuropsychopharmacology 22, 108-124 (1984).

18) Charney DS: Psychobiological mechanisms of resilience and vulnerability: implications for successful adaptation to extreme stress. Am J Psychiatry 161, 195-216 (2004).

19) Sakamoto M, Higaki Y, Nishida Y, Kiyonaga A, Shindo M, Tokuyama K, et al.: Influence of mild exercise at the lactate threshold on glucose effectiveness. J Appl Physiol 87, 2305-2310 (1999). 